\title{
Risk factors and risk reduction of breast and ovarian cancer Cecile T.M. Brekelmans
}

\section{Purpose of review}

Breast and ovarian cancer remain a significant burden for women living in the Western world. This paper reviews the risk factors and current strategies to prevent these diseases.

\section{Recent findings}

Established factors associated with the risk of breast cancer include family history, reproductive factors and lactation, as well as age at menarche and menopause. Hormone replacement therapy increases the risk, whereas oral contraceptives probably confer no increased risk. Alcohol moderately increases the risk, whereas a diet rich in folate and carotenoids might be protective. The role of other dietary factors, smoking and physical exercise remain unclear. Important risk factors for ovarian cancer are reproductive factors and possibly the longterm use of hormone replacement therapy. The risk is decreased by oral contraceptives. In carriers of a BRCA1 or BRCA2 gene mutation, prophylactic surgery can significantly reduce the risk of breast as well as ovarian cancer. Tamoxifen may be considered as a chemopreventive agent in women with a high risk of breast cancer, including carriers of a BRCA2 mutation, but is probably not effective in BRCA1 carriers.

\section{Summary}

During the period of this review, the importance of several known risk factors was confirmed, whereas the effects of other factors became more clear. Chemoprevention and prophylactic surgery have emerged as preventative options that can reduce the risk of breast and ovarian cancer.

\section{Keywords}

breast cancer, ovarian cancer, prevention, review, risk factors

Curr Opin Obstet Gyneol 15:63-68. () 2003 Lippincott Williams \& Wilkins.

Department of Medical Oncology, Erasmus MC - Daniel den Hoed, Rotterdam, the Netherlands

Correspondence to Cecile T.M. Brekelmans, MD, PhD, Department of Medical Oncology, Erasmus MC - Daniel den Hoed, P.O. Box 5201, 3008 AE Rotterdam, the Netherlands

Tel: +31 104391633; fax: +31 104391003; e-mail: brekelmans@onch.azr.nl

Current Opinion in Obstetrics and Gynecology 2003, 15:63-68

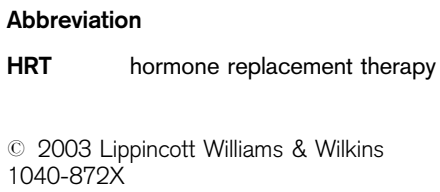

\section{Introduction}

This paper reviews recent studies (mainly published since July 2001) on risk factors and risk-reducing strategies for breast and ovarian cancer. The effectiveness of screening for breast and ovarian cancer is not considered in this review and will be discussed elsewhere.

\section{Breast cancer}

Breast cancer remains the most frequent type of cancer in women in the world with nearly 1 million new cases each year worldwide. In most developed countries the incidence levels are levelling off and, very recently, declining in some countries, especially in women under the age of 40 years [1].

With a total of 375000 deaths each year, it is also the leading cause of cancer mortality in Europe, and the second in the United States only after lung cancer [2,3]. Mortality figures have been declining for a number of years now, and this trend continues, most likely by a combination of early detection and improved treatment [3].

\section{Risk factors}

Breast cancer is a hormonally related process; most breast cancer risk factors act through changes in hormonal (mainly oestrogen) levels that influence the breast epithelium.

\section{Familial breast cancer}

Family history is the most important breast cancer risk factor. Approximately $5 \%$ of all breast cancers are caused by germline mutations in the BRCA1 and BRCA2 genes.

Evidence of a third breast cancer predisposition gene was found by a combined investigation of Dutch and British researchers. They found that mutations in CHEK-2, a gene involved in DNA repair, account for approximately $1 \%$ of all breast cancers. The clinical application is still limited as segregation analysis suggests that the mutation is only expressed in combination with another, as yet unknown, gene mutation $\left[4^{\bullet \bullet}\right]$.

Furthermore, two recurrent $A T M$ mutations might be associated with a nearly 16 -fold increased risk compared with the general population $\left[5^{\bullet \bullet}\right]$.

Other genetic and environmental factors probably further modify the risk. A recent review [6] found an 
association for 13 polymorphisms in 10 genes. In addition, findings from a genetic modelling study [ $\left.7^{\bullet}\right]$ suggested that several low-penetrance genes rather than one ' $B R C A 3$ ' gene account for the residual familial aggregation of breast cancer.

\section{Reproductive factors}

Age at first childbirth, parity and age at menarche are well-known factors that are associated with the risk of breast cancer. Their strength varies with age at diagnosis [8].

Placental characteristics might play a role: a large followup study [ $\left.9^{\circ}\right]$, which started more than 40 years ago, provided evidence that smaller placentas, maternal floor infarction and increasing blood pressure during pregnancy all reduced the risk of breast cancer. The protective effect of pre-eclampsia, another marker of compromised placental function, was already known. A case-control study in 66 pregnancies with severe preeclampsia and 610 controls [10] suggested that the possible mechanism might be high levels of alphafetoprotein rather than low levels of oestradiol.

A history of induced abortion appears to have little influence on the risk of breast cancer, at least in Chinese women [11].

The effect of breast feeding has long been unclear but now appears to be firmly established: in addition to several individual studies, a collaborative re-analysis of data from 47 studies with a total of 50302 women with breast cancer and 96973 healthy women showed that the relative risk of breast cancer decreased by $4.3 \%$ for each year of breast feeding [12॰].

\section{Oral contraceptives}

The risk of oral contraceptive usage appeared to be clearly established, with numerous case-control and cohort studies showing a small increased risk of breast cancer with long-term usage of oral contraceptives, especially at a young age. However, a very large study was recently published that found no association between current or past use of oral contraceptives and breast cancer. The study was, with 4575 breast cancer cases and 4682 controls, large enough to perform subgroup analyses with respect to long duration of use, a high dosage of oestrogens, initation at a young age, or a family history of breast cancer. None of these subgroups had a significantly increased risk $\left[13^{\bullet \bullet}\right]$.

\section{Hormone replacement therapy}

Much publicity was gained by the publication of the Women's Health Initiative (WHI) randomized controlled trial, in which women were randomly assigned between receiving oestrogens plus medroxyprogester- one acetate $(n=8506)$ or placebo $(n=8102)$. In addition to an excess of cardiovascular disease, the risk of breast cancer was increased in the treatment arm [hazard ratio (HR) 1.27 ; $95 \%$ confidence interval (CI) 1.00-1.59]. The trial was terminated early $\left[14^{\bullet \bullet}\right.$. The results are consistent with the (non-significant) 27\% increase found in the Heart and Estrogen Replacement Study II in postmenopausal women with coronary disease [15] and estimates from numerous observational studies suggesting an increased risk, especially of current longterm use ( $>5$ years).

\section{Benign breast disease}

The assocation between proliferative breast lesions, especially atypical hyperplasia, and breast cancer has long been recognized. The risk of breast cancer in patients with atypical hyperplasia is approximately equal in both breasts. Furthermore, the pathological features of breast cancers developing in women with benign breast disease, including atypical hyperplasia, do not vary according to the histology of the previous benign breast lesion [16]. Together, these findings suggest that these lesions are risk indicators rather than direct precursors of breast cancer.

\section{Alcohol and diet}

In agreement with many previous studies, a large prospective study with 1328 incident cases of breast cancer found that alcohol use moderately increased the risk of breast cancer [17]. Although the assocation with the 'western' diet (characterized by meat, fat, dairy and sweets) remains unconfirmed [17,18], a number of other factors are now consistently associated with the risk of breast cancer; carotenoids decrease the risk [19] as well as folate [20]. The risk of low folate intake is more increased in combination with alcoholic beverages [21].

A decreased risk of soy (phytoestrogens) has only been established in Chinese women; a case-control study in Shanghai [22] suggested that regular soy consumption reduced the risk of breast cancer, especially for ER/PR-positive tumours. In non-Asian women phytoestrogens appeared to have little effect [23].

\section{Smoking}

Until recently, studies into the role of active and passive smoking failed to show an effect of smoking on the risk of breast cancer. It might be that the contrast in the comparison of ever versus never smokers was insufficient. Data from the Nurses' Health Study were compatible with a small increased risk of active smoking, with the highest risks in women initiating smoking before the age of 17 years. No association with passive smoking was found [24]. 


\section{Anthropometry}

It is well known that height and weight are related to breast cancer. The assocation with weight differs according to menopausal status. Whereas leanness is positively associated with premenopausal breast cancer, overweight (especially visceral) increases the risk of postmenopausal breast cancer. This was confirmed in a Canadian case-control study of 1233 incident breast cancer cases and 1241 controls: a larger waist-hip ratio and weight gain increase the risk of postmenopausal breast cancer [25]. Both a Finnish cohort study in 3447 women and a pooled analysis of four case-control studies $[26,27]$ suggested that the risk of premenopausal breast cancer is affected early in life; a positive association was found for tallness during childhood whereas a negative association was found with body mass index at a young age.

\section{Physical activity}

The evidence remains inconsistent; a case-control study of 1237 incident cases and 1241 controls suggested that sustained physical activity throughout life and particularly later in life moderately reduced the risk of breast cancer [28]. On the other hand, a case-control study of 394 cases and 788 controls showed no assocation with lifetime physical activity [29]. The reasons for this inconsistency might be different assessment methods of physical activity or interaction with other factors. This is suggested by a Swedish cohort study [30] that found an association with physical activity only for normal-weight postmenopausal and overweight premenopausal women.

\section{Environmental factors}

A Canadian case-control study found an association with dioxin-like polychlorinated biphenyls, suggesting that exposure to these substances might increase the risk of breast cancer or, alternatively, that cases and controls have different metabolic pathways involved in the biotransformation of polychlorinated biphenyls and oestrogens [31]. P53 mutations might modify this association [32].

Two studies, a case-control and a cohort $\left[33^{\bullet}, 34^{\bullet}\right]$, were published that found a positive association between the disruption of the diurnal sleep-wakefulness rhythm, especially during night-shift work and the risk of breast cancer. The mechanism is unclear but is postulated to be exposure to light at night, causing a reduced level of melatonin, a hormone that suppresses ovarian oestrogen production.

Chronic exposure to residential magnetic fields might also suppress the nocturnal production of melatonin. However, no assocation was found in a recent casecontrol study with 813 cases and 793 age-matched controls [35].

\section{Ovarian cancer}

Epithelial ovarian cancer is the seventh most frequent cancer in European women, with 58000 new cases and 38000 deaths in 1995 [36]. The incidence is low under the age of 40 years but increases rapidly after menopause. Despite new chemotherapy agents, the prognosis remains fairly poor with a 5 -year relative survival of $30 \%$ [37].

\section{Risk factors}

Many theories have been postulated regarding the pathogenesis of ovarian cancer. Risk factors are not well defined, with the exception of parity and oral contraceptive use. Most studies consider the epidemiology of epithelial ovarian cancer, representing approximately $90 \%$ of all ovarian carcinomas.

\section{Hereditary ovarian cancer}

Approximately $10 \%$ of ovarian cancers are hereditary, with BRCA1 and BRCA2 explaining the majority (approximately 90\%) of hereditary ovarian cancer cases. The lifetime risk varies between 15 and 66\%, suggesting the existence of modifying genetic or environmental factors [38].

\section{Reproductive factors}

The protective effects of increasing parity and breast feeding have been clearly established. Early age at menarche and late age at menopause increase the risk of ovarian cancer only modestly, so that it can be assumed that the length of menstrual life plays no crucial role in the pathogenesis of the disease [39].

The risk of sub/infertility on the risk of ovarian cancer has been extensively studied. Most studies use attendance at an infertility clinic as a marker for subfertility. The difficulty is the differentiation between the infertility itself and the treatment given at the clinic, that may in itself increase the risk of ovarian cancer. A pooled analysis of case-control studies, including 5207 cases and 7705 controls, suggested that specific biological causes of infertility rather than fertility drugs increased the risk of ovarian cancer $\left[40^{\bullet}\right]$.

\section{Oral contraceptives}

Several studies have consistently shown a decreased risk of sporadic as well as familial ovarian cancer [41]. The risk reduction is already apparent after a few months of use and persists for years after discontinuation. Lowdose formulations $(<35 \mu \mathrm{g}$ ethinyl oestradiol $)$ also confer a substantial risk reduction [42]. However, inconsistent results were reported for women at risk of hereditary ovarian cancer. Although a case-control study in 207 BRCA1/2 carriers [43] reported a significant $50 \%$ risk reduction, a case-control study in Jewish women $\left[44^{\bullet}\right]$ found that oral contraceptives significantly 
decreased the risk of ovarian cancer only in non-carriers but not in carriers of a BRCA1/2 mutation.

\section{Oestrogen/hormone replacement therapy}

Hormone replacement therapy (HRT) has been inconsistently linked to ovarian cancer. Recently, several prospective studies $\left[45^{\circ}, 46^{\circ}\right]$ consistently found a small increased risk, especially for long-term users of oestrogen replacement therapy. Sequentially rather than continuously added progestins might further increase the risk [47].

\section{Diet}

The evidence remains inconsistent; recent studies suggest that food items high in carotene and lycopene might decrease the risk [48], whereas red meat was associated with an elevated risk [49]. However, all positive studies were case-control studies, and a recent prospective study within the Nurses' Health Study found no association between the usage of vitamins/ carotenoids and ovarian cancer [50]. It might be that (part of) the reported associations in previous studies were caused by the various types of bias (especially recall bias) that can occur in case-control studies.

\section{Physical activity}

It is hypothesized that recreational physical activity may reduce the risk of ovarian cancer by decreasing oestrogen levels. However, although this reduced risk was found by several studies, others found no or even a modest positive assocation. The most recent study [51], with 327 cases and 3129 controls, reported a small, non-significant, decrease in risk only for the highest category of recent vigorous activity.

\section{Use of non-steroidal anti-inflammatory drugs}

Non-steroidal anti-inflammatory drugs increase apoptosis in ovarian cancer cell lines and can inhibit ovulation [52]. In observational studies, the association between commonly used anti-inflammatory drugs and ovarian cancer remains unclear. A Danish cohort study found no evidence of a protective effect of paracetamol [53], and a case-control study conducted in the USA concluded that, although non-significant, the observed risk estimates appeared to be compatible with a small decrease of risk by regular (more than three times a week for a period of at least 6 months) aspirin use [54].

\section{Risk reduction}

Options to reduce the risk of breast and ovarian cancer include life-style modification, prophylactic surgery and chemoprevention.

\section{Life-style modification}

Risk factors that are most amenable for primary prevention are dietary factors (including alcohol), smok- ing and physical exercise. Although the impact on the risk of breast and ovarian cancer is either unclear or at best modest, a healthy life-style with the avoidance of smoking, moderate usage of alcohol, and physical exercise is advisable as it might also decrease the risk of a number of other diseases, such as other cancer types and cardiovascular disease.

\section{Prophylactic surgery}

The short-term potential of prophylactic mastectomy was clearly demonstrated by a prospective study in 139 BRCA1/2 carriers. Within 3 years' follow-up, no cases of breast cancer appeared in 76 women opting for prophylactic mastectomy and eight occurred in 63 women opting for intensive surveillance $\left[55^{\bullet \bullet}\right]$.

Two complementary studies in $B R C A 1 / 2$ gene mutation carriers demonstrated that prophylactic (salpingo-)oophorectomy can reduce the risk of hereditary ovarian as well as breast cancer. In a prospective study of 170 carriers with a mean follow-up of 2 years [56 $\left.{ }^{\bullet}\right]$, one woman developed peritoneal cancer and three breast cancer in the salpingo-oophorectomy group of 98 women. Three occult stage I gynaecological tumours were found at the time of prophylactic surgery. In the surveillance group of 72 women, five women developed ovarian or peritoneal cancer and eight developed breast cancer. The combined HR for breast and gynaecological cancer was 0.25 (95\% CI 0.08-0.74).

In a multicentre retrospective study with a mean followup of almost 9 years [57 ${ }^{\bullet}$ ], 58 out of 292 carriers opting for surveillance developed ovarian cancer. Among 259 women undergoing prophylactic oophorectomy, two women developed primary peritoneal cancer (HR 0.04; 95\% CI 0.01-0.16). In six women a stage I ovarian cancer was diagnosed at the time of surgery. Breast cancer was diagnosed in 60 out of 142 women opting for surveillance compared with 21 out of 99 women in the oophorectomy group, giving an HR of 0.47 (95\% CI 0.29-0.77).

The risk-reducing potential of tubal ligation had already been shown in sporadic ovarian cancer. In addition, a matched case-control study in 232 BRCA1/2 carriers with ovarian cancer and 232 carriers without the disease [58] showed that the adjusted odds ratio of developing ovarian cancer was $0.39(P=0.002)$ after tubal ligation. The biological mechanism is unclear; hypotheses include the reduction of ovarian blood supply or a decreased retrograde transport of potential carcinogens through the fallopian tubes.

\section{Breast cancer chemoprevention}

The effect of tamoxifen in reducing the short-term risk of oestrogen receptor-positive tumours is clearly estab- 
lished. A meta-analysis including updated results of all four randomized trials showed a significant $38 \%$ reduction in the incidence of breast cancer [59]. Its chemopreventive potential in $B R C A 1$ mutation carriers, at increased risk of oestrogen receptor-negative tumours, is less clear. In a subgroup analysis of $19 \mathrm{BRCA} 1 / 2$ cases participating in the National Surgical Adjuvant Breast and Bowel Project Breast Cancer Prevention Trial (NSABP-P1), no reduction of breast cancer incidence in BRCA1 carriers was observed [relative risk (RR) 1.67; 95\% CI 0.41-8.0], whereas there was a suggestion of a benefit in BRCA2 carriers (RR 0.38; 95\% CI 0.06-1.56) $\left[60^{\circ}\right]$. The small number of cases warrants replication in larger datasets. A modelling study estimating the potential impact of tamoxifen on $B R C A 1 / 2$ carriers [61] concluded that the benefit of tamoxifen is likely to be modest in BRCA1 carriers and might be larger in BRCA2 carriers. Future chemopreventive agents may include raloxifene, which is now being compared with tamoxifen in the STAR trial [59].

\section{Ovarian cancer chemoprevention}

Oral contraceptives substantially reduce the risk of sporadic ovarian cancer, so might be an effective chemopreventive agent in high-risk women. However, because of the inconsistent results in $B R C A 1 / 2$ carriers $\left[43,44^{\circ}\right]$, it is premature to consider oral contraceptives for the chemoprevention of hereditary ovarian cancer.

\section{Conclusion}

In the period covered in this review, the importance of several known risk factors for breast and ovarian cancer was confirmed, whereas the effects of lactation, some dietary factors and HRT became more clear. In contrast to previous concerns, oral contraceptives probably confer no increased risk of breast cancer.

The publication of several prospective studies on the potential of risk-reducing surgery in BRCA1/2 carriers provides evidence facilitating decision-making on preventative strategies in these high-risk women.

Whereas tamoxifen and oral contraceptives may be considered as chemopreventive agents for the reduction of the risk of breast and ovarian cancer, respectively, their potential in reducing the risk of hereditary breast and ovarian cancer is still unclear.

\section{References and recommended reading}

Papers of particular interest, published within the annual period of review, have been highlighted as:

- of special interest

- of outstanding interest

1 Lacey JV, Devesa S, Brinton LA. Recent trends in breast cancer incidence and mortality. Environ Mol Mutagen 2002; 39:82-88.
2 Jemal A, Thomas A, Murray T, Thun M. Cancer statistics, 2002. CA Cancer J Clin 2002; 52:23-47.

3 Parkin DM, Bray FI, Devesa SS. Cancer burden in the year 2000. The global picture. Eur J Cancer 2001; 37 (Suppl):4-66.

4 The CHEK-2-Breast Cancer Consortium. Low-penetrance susceptibility to -. breast cancer due to CHEK2100delC in noncarriers of BRCA1 or BRCA2 mutations. Nat Genet 2002; 31:55-59.

This is the first study that finds evidence for a third breast cancer predisposition gene: mutations in CHEK-2 (also known as CHK-2), a gene involved in DNA repair, may account for approximately $1 \%$ of all breast cancers.

5 Chenevix-Trench G, Spurdle A, Gatei M, et al. Dominant negative ATM -. mutations in breast cancer families. J Natl Cancer Inst 2002; 94:205-215.

The first study demonstrating the high breast cancer risk of two recurrent ATM mutations, implying that genetic screening for ATM mutations may be worthwile.

6 De Jong MM, Nolte IM, te Meerman, et al. Genes other than BRCA1 and BRCA2 involved in breast cancer susceptibility. J Med Genet 2002; 39:225242.

7 Antoniou AC, Pharoah PDP, McMullan G, et al. A comprehensive model for - familial breast cancer incorporating BRCA1, BRCA2 and other genes. $\mathrm{Br} J$ Cancer 2002; 86:76-83.

A segregation study investigating the genetic model that can best explain the residual non-BRCA1/2 familial aggregation of breast cancer.

8 Tryggvadottir L, Tulinius H, Eyfjord JE, Sigurvinsson T. Breast cancer risk factors and age at diagnosis: an Icelandic cohort study. Int J Cancer 2002; 98:604-608.

9 Cohn BA, Cirillo PM, Christianson RE, et al. Placental characteristics and - reduced risk of maternal breast cancer. J Natl Cancer Inst 2001; 93:11331140.

A cohort study with 40 years of follow-up that increases our understanding of the biology underlying the reduction in breast cancer risk by parity and age at first fullterm pregnancy.

10 Vatten LJ, Romundstad PR, Odegard RA, et al. Alpha-foetoprotein in umbilical cord in relation to severe pre-eclampsia, birth weight and future breast cancer risk. Br J Cancer 2002; 86:728-731.

11 Sanderson $\mathrm{M}$, Shu $\mathrm{XO}$, Jin $\mathrm{F}$, et al. Abortion history and breast cancer risk: results from the Shanghai Breast Cancer Study. Int J Cancer 2001; 92:899905.

12 Collaborative Group on Hormonal Factors in Breast Cancer. Breast cancer - and breastfeeding: collaborative reanalysis of individual data from 47 epidemiological studies in 30 countries, including 50302 women with breast cancer and 96973 women without the disease. Lancet 2002; 360:187-195.

A large collaborative re-analysis of 47 case-control and cohort studies quantifying the protective effect of breast feeding on the risk of breast cancer, taking account of other reproductive factors.

13 Marchbanks PA, McDonald JA, Wilson HG. Oral contraceptives and the risk -. of breast cancer. N Engl J Med 2002; 346:2025-2032.

A large single study in 4575 women with breast cancer and 4682 controls between 35 and 64 years of age. The study was large enough to perform subgroup analyses with respect to race, age at initiation, duration and dosage of use and family history of breast cancer.

14 Writing Group for the Women's Health Initiative Investigators. Risks and

- benefits of estrogen plus progestin in healthy postmenopausal women. JAMA 2002; 288:321-333.

The first randomized controlled trial that assessed the benefits and risks of HRT in healthy women.

15 Hulley S, Furberg C, Barrett-Connor E, et al., for the HERS Research Group. Non-cardiovascular disease outcomes during 6.8 years of hormone therapy: Heart and Estrogen Replacement Study (HERS II) follow-up. JAMA 2002; 288:58-66.

16 Jacobs TW, Byrne C, Colditz G, et al. Pathologic features of breast cancers in women with prior benign breast disease. Am J Clin Pathol 2001; 115:362369.

17 Terry $\mathrm{P}$, Suzuki R, Hu FB, Wolk A. A prospective study of major dietary patterns and the risk of breast cancer. Cancer Epidemiol Biomarkers Prev 2001; 10:1281-1285.

18 Missmer SA, Smith-Warner SA, Spiegelman D, et al. Meat and dairy food consumption and breast cancer: a pooled analysis of cohort studies. Int J Epidemiol 2002; 31:78-85.

19 Toniolo P, Van Kappel AL, Akhmedkhanov A, et al. Serum carotenoids and breast cancer. Am J Epidemiol 2001; 153:1142-1147.

20 Shrubsole MJ, Jin F, Dai Q, et al. Dietary folate intake and breast cancer risk: results from the Shanghai Breast Cancer Study. Cancer Res 2001; 61:136141. 
21 Sellers TA, Kushi LH, Cerhan JR, et al. Dietary folate intake, alcohol, and risk of breast cancer in a prospective study of postmenopausal women. Epidemiology 2001; 12:420-428.

22 Dai Q, Shu XO, Jin F, et al. Population-based case-control study of soyfood and breast cancer risk in Shanghai. Br J Cancer 2001; 85:372-378.

23 Horn-Ross PL, John EM, Lee $M$, et al. Phytoestrogen consumption and breast cancer risk in a multiethnic population: the Bay Area Breast Cancer Study. Am J Epidemiol 2001; 154:434-441.

24 Egan KM, Stampfer MJ, Hunter D, et al. Active and passive smoking in breast cancer: prospective results from the Nurses' Health Study. Epidemiology 2002; 13:138-145.

25 Friedenreich CM, Courneya KS, Bryant HE. Case-control study of anthropometric measures and breast cancer risk. Int J Cancer 2002; 99:445-452.

26 Hilakivi-Clarke L, Forsen T, Eriksson JG, et al. Tallness and overweight during childhood have opposing effects on breast cancer risk. $\mathrm{Br} \mathrm{J}$ Cancer 2001; 85:1680-1684.

27 Swerdlow AJ, De Stavola BL, Floderus B, et al. Risk factors for breast cancer at young ages in twins: an international population-based study. J Natl Cancer Inst 2002; 21:1238-1246.

28 Friedenreich CM, Courneya KS, Bryant HE. Influence of physical activity in different age and life periods on the risk of breast cancer. Epidemiology $2001 ; 12: 604-612$.

29 Lee IM, Cook NR, Rexrode KM, Buring JE. Lifetime physical activity and risk of breast cancer. Br J Cancer 2001; 85:962-965.

30 Moradi T, Adami HO, Eckbom A, et al. Physical activity and risk for breast cancer: a prospective cohort study among Swedish twins. Int J Cancer 2002; 100:76-81.

31 Demers A, Ayotte P, Brisson J, et al. Plasma concentrations of polychlorinated biphenyls and the risk of breast cancer: a congener-specific analysis. Am J Epidemiol 2002; 155:629-635.

32 Hoyer AP, Gerdes AM, Jorgensen T, et al. Organochlorines, p53 mutations in relation to breast cancer risk and survival. A Danish cohort-nested casecontrol study. Breast Cancer Res Treat 2002; 71:59-65.

33 Davis S, Mirick DK, Stevens RG. Night shift work, light at night and risk of - breast cancer. J Natl Cancer Inst 2001; 93:1557-1562.

A case-control study into the relationship between light at night and breast cancer, with detailed exposure information including sleeping habits, bedroom environment and graveyard shiftwork.

34 Schernhammer ES, Laden F, Speizer FE, et al. Rotating night shifts and risk - of breast cancer in women participating in the Nurses' Health Study. J Natl Cancer Inst 2001; 93:1563-1568.

A large cohort study with long follow-up into the relationship between rotating night shifts with at least three nights per month and breast cancer.

35 Davis S, Mirick DK, Stevens RG. Residential magnetic fields and the risk of breast cancer. Am J Epidemiol 2002; 155:446-454.

36 Bray F, Sankila R, Ferlay J, Parkin DM. Estimates of cancer incidence and mortality in Europe in 1995. Eur J Cancer 2002; 38:99-166.

37 Berrino F, Capocaccia R, Esteve J, et al. Survival of cancer patients in Europe: The Eurocare-2 Study. IARC scientific publication no. 151. Lyon: IARC; 1999

38 Antoniou AC, Gayther SA, Stratton J, et al. Risk models for familial ovarian and breast cancer. Genet Epidemiol 2000; 18:173-190.

39 Schildkraut JM, Cooper GS, Halabi S, et al. Age at natural menopause and the risk of epithelial ovarian cancer. Obstet Gynaecol 2001; 98:85-90.

40 Ness RB, Cramer DW, Goodman MT, et al. Infertility, infertility drugs and ovarian cancer: a pooled analysis of case-control studies. Am J Epidemiol 2002; 155:217-224.

A pooled analysis of eight case-control studies into the association between sub/ infertility and ovarian cancer that was able to differentiate between biological causes of infertility and the use of fertility drugs.

41 Walker GR, Schlesselman JJ, Ness RB. Family history of cancer, oral contraceptive use, and ovarian cancer risk. Am J Obstet Gynecol 2002; 186:8-14.

42 Royar J, Becher H, Chang-Claude J. Low-dose oral contraceptives: protective effect on ovarian cancer risk. Int J Cancer 2001; 95:370-374.

43 Narod SA, Risch $\mathrm{H}$, Moslehi $\mathrm{R}$, et al. Oral contraceptives and the risk of hereditary ovarian cancer. N Engl J Med 1998; 339:424-428.
44 Modan B, Hartge P, Hirsch-Yechezkel G, et al. Parity, oral contraceptives, and the risk of ovarian cancer among carriers and noncarriers of a BRCA1 or BRCA2 mutation. N Engl J Med 2001; 345:235-240.

A population-based case-control study investigating the association between oral contraceptive use and the risk of ovarian cancer, differentiating between carriers and non-carriers of a BRCA1 or BRCA2 mutation.

45 Rodriguez C, Patel AV, Calle EE, et al. Estrogen replacement therapy and

- ovarian cancer mortality in a large prospective study of US women. JAMA $2001 ; 285: 1460-1465$

A large, prospective study with long follow-up investigating the association between oestrogen replacement therapy and ovarian cancer mortality in postmenopausal women.

46 Lacey JV, Mink PJ, Lubin JH, et al. Menopausal hormone replacement - $\quad$ therapy and risk of ovarian cancer. JAMA 2002; 288:334-341.

A large cohort study into the risk of ovarian cancer after menopausal hormone therapy, differentiating between oestrogen-only and oestrogen-progestin formulas.

47 Riman T, Dickman PW, Nilsson S, et al. Hormone replacement therapy and the risk of invasive epithelial ovarian cancer in Swedish women. J Natl Cancer Inst 2002; 94:497-504.

48 Cramer DW, Kuper H, Harlow BL, Titus-Ernstoff L. Carotenoids, antioxidants and ovarian cancer risk in pre- and postmenopausal women. Int J Cancer 2001; 94:128-134.

49 Bosetti C, Negri E, Franchesci S, et al. Diet and ovarian cancer risk: a casecontrol study in Italy. Int J Cancer 2001; 93:911-915.

50 Fairfield KM, Hankinson SE, Rosner BA, et al. Risk of ovarian carcinoma and consumption of vitamins $A, C$, and $E$ and specific carotenoids: a prospective analysis. Cancer 2001; 92:2318-2326.

51 Bertone ER, Newcomb PA, Willett WC, et al. Recreational physical activity and ovarian cancer in a population-based case-control study. Int J Cancer 2002; 99:431-436

52 Rodriguez-Burford C, Barnes MN, Oelschlager DK, et al. Effects of nonsteroidal anti-inflammatory agents (NSAIDs) on ovarian carcinoma cell lines: preclinical evaluation of NSAIDs as chemopreventive agents. Clin Cancer Res 2002; 8:202-209.

53 Friis S, Nielsen GL, Mellemkjaer L, et al. Cancer risk in persons receiving prescriptions for paracetamol: a Danish cohort study. Int J Cancer 2002; 97:96-101.

54 Akhmedkhanov A, Toniolo $\mathrm{P}$, Zeleniuch-Jacquotte $\mathrm{A}$, et al. Aspirin and epithelial ovarian cancer. Prevent Med 2001; 33:682-687.

55 Meijers-Heijboer H, van Geel B, van Putten WLJ, et al. Breast cancer after

-. prophylactic bilateral mastectomy in women with a BRCA1 or BRCA2 mutation. N Engl J Med 2001; 345:159-164.

The first prospective evaluation of the risk of breast cancer after prophylactic mastectomy in confirmed carriers of a BRCA1 or BRCA2 gene mutation, al ascertained in one family cancer clinic.

56 Kauff ND, Satagopan JM, Robson ME, et al. Risk-reducing salpingo-

- oophorectomy in women with a BRCA1 or BRCA2 mutation. N Engl J Med 2002; 346:1609-1615

The first prospective evaluation in 170 BRCA1/2 carriers investigating the incidence of breast and ovarian cancer after risk-reducing salpingo-oophorectomy

57 Rebbeck TR, Lynch HT, Neuhausen SL, et al. Prophylactic oophorectomy in

- carriers of BRCA1 or BRCA2 mutations. N Engl J Med 2002; 346:16161622.

A retrospective study with long follow-up in 551 BRCA1/2 carriers identified from 11 North American and European registries investigating the incidence of ovarian cancer after prophylactic oophorectomy.

58 Narod SA, Sun $\mathrm{P}$, Ghadirian $\mathrm{P}$, et al. Tubal ligation and risk of ovarian cancer in carriers of BRCA1 or BRCA2 mutations: a case-control study. Lancet 2001; 357:1467-1470.

59 Chlebowski RT, Col N, Winer EP, et al. American Society of Clinical Oncology technology assessment of pharmacologic interventions for breast cancer risk reduction including tamoxifen, raloxifene, and aromatase inhibition. J Clin Oncol 2002; 20:3328-3343.

60 King MC, Wieand S, Hale K, et al. Tamoxifen and breast cancer incidence among women with inherited mutations in BRCA1 and BRCA2. JAMA 2001; 286:2251-2256.

Thr first study addressing the chemopreventive effect of tamoxifen in confirmed

BRCA1 and BRCA2 carriers participating in the NSABP-P1 Breast Cancer Prevention Trial.

61 Duffy SW, Nixon RM. Estimates of the likely prophylactic effect of tamoxifen in women with high risk BRCA1 and BRCA2 mutations. Br J Cancer 2002 86:218-221. 